\title{
Mortalidade por doença cardíaca hipertensiva nas macrorregiões brasileiras
}

\author{
Mortality by hypertensive heart disease in Brazilian macroregions
}

\author{
Amanda Silva Fragaa ${ }^{a}$ Cloud Kennedy Couto de Sáb, Mário César Carvalho Tenório \\ a Aluna de graduação do Curso de Medicina, Escola Bahiana de Medicina e Saúde Pública, Bahia, Brasil. \\ b Educador Físico. Doutor em Medicina e Saúde Humana pela Escola Bahiana de Medicina e Saúde Pública. Professor da Universidade Estadual de Feira de Santana, Bahia, Brasil. \\ c Educador Físico. Mestre em Medicina e Saúde Humana pela Escola Bahiana de Medicina e Saúde Pública. Professor da Faculdade Social da Bahia, Bahia, Brasil.
}

Introdução: As doenças cardiovasculares estão em primeiro lugar entre as causas de morte no Brasil. A doença cardíaca hipertensiva ( $\mathrm{DCH}$ ) descreve um espectro de resposta de órgãos-alvo, que inclui doença arterial coronariana, hipertrofia ventricular esquerda, insuficiência cardíaca e arritmias.

Objetivo: Realizar uma análise epidemiológica da mortalidade por DCH nas macrorregiões brasileiras.

Materiais e Métodos: Estudo descritivo das taxas de mortalidade de base populacional com dados secundários e uma análise comparativa entre as macrorregiões brasileiras, utilizando as variáveis: sexo, faixa-etária, ano do óbito, cor/raça e escolaridade.

Resultados: Observou-se grande acréscimo nos coeficientes de mortalidade brutos a partir de 40 a 59 anos. A DCH acometeu principalmente pessoas pardas nas regiões norte, nordeste e centro-oeste e indivíduos brancos nas regiões sul e sudeste, provavelmente por maior predomínio desses fenótipos nas respectivas áreas. Quanto ao sexo, a mortalidade é elevada em ambos. O número de óbitos é inversamente proporcional à quantidade de anos de escolaridade em todas as regiões, sendo os maiores valores no grupo de analfabetos.

Conclusão: Observamos que com o aumento da idade há elevação do coeficiente de mortalidade, especialmente onde há maior precariedade na assistência à saúde, nas medidas de prevenção, controle e acompanhamento, além de repasse de recursos, a exemplo da região nordeste.

Palavras-chave: doença cardíaca hipertensiva; hipertensão; epidemiologia. describes a target-organ response spectrum, which includes coronary artery disease, left ventricular hypertrophy, heart failure and arrhythmias.

Objective: To carry out an epidemiological analysis of mortality by HHD in the Brazilian regions.

Materials and Methods: Descriptive population-based study of mortality rates with secondary data, as well as comparative analysis between the Brazilian regions using the following variables: gender, age group, year of death, color/race and education.

Results: There was a large increase in crude mortality rates from 40 to 59 years. HHD occurred mainly in brown people in the North, Northeast and Midwest, and whites in the South and Southeast regions, probably due to greater predominance of these phenotypes in their respective areas. Regarding sex, mortality was high in both. The number of deaths is inversely proportional to the number of years of schooling in all regions, with the highest values in the illiterate group.

Conclusion: We have observed that, as age increases, there is an elevated mortality rate, especially where there is a greater insecurity in health care, control, follow-up and preventive measures, as well as in mechanisms of funding transfer, such as in the Northeast.

Keywords: hypertensive heart disease; hypertension; epidemiology. 


\section{INTRODUÇÃO}

As doenças cardiovasculares estão em primeiro lugar entre as causas de morte no Brasil. Representam um terço dos óbitos totais, $65 \%$ do total de mortes nos indivíduos de 30 a 69 anos de idade e atinge 32,5\% dos adultos, acometendo a população em plena fase produtiva ${ }^{1,2}$.

Entre as doenças cardiovasculares, a insuficiência cardíaca é aquela cujos estudos epidemiológicos são mais difíceis de serem realizados, pois há uma diversidade de definições e de critérios vigentes sem consenso, além de múltiplas etiologias e exames diagnósticos específicos, tornando os estudos de elevado custo ${ }^{3}$.

A hipertensão arterial sistêmica (HAS) figura entre as doenças mais prevalentes em nosso país. Segundo o Ministério da saúde, a HAS atinge cerca de $30 \%$ dos brasileiros adultos, sendo determinante de alta morbidade e um dos principais fatores de risco para as doenças cerebrovasculares e cardiovasculares. Mesmo quando não causam a morte, suas complicações podem levar a invalidez parcial ou total, o que torna sua triagem e acompanhamento imprescindíveis em virtude dos danos gerados, que são responsáveis por elevados custos médico-hospitalares,onerando o sistema público e à sociedade ${ }^{4}$.

A resistência ao tratamento, usualmente acompanha-se de maior lesão aos órgãos-alvo (retina, coração, cérebro e rim). Identificá-las segundo o risco e progressão das lesões vasculares e determinar a gravidade da HAS e sua resistência ao tratamento é fundamental, pois estas lesões repercutem em funcionalidade e qualidade de vida, especialmente quando há interação com comorbidades, se associando ao aumento da morbidade e mortalidade e acréscimo do risco cardiovascular ${ }^{5}$.

Dentre as complicações cardíacas secundárias ao aumento persistente da pressão arterial, temos a doença cardíaca hipertensiva (DCH) ou cardiopatia hipertensiva, que é a primeira causa cardiovascular de hospitalização, muito frequente nos serviços de emergência. A DCH descreve um espectro de resposta de órgãos-alvo, que inclui doença arterial coronariana, hipertrofia ventricular esquerda, insuficiência cardíaca, dilatação ventricular, fibrilação atrial e outras arritmias cardíacas e a morte súbita ${ }^{6-8}$. A hipertrofia do ventrículo esquerdo é uma das complicações cardíacas da HAS, tendo prevalência em torno de $40 \%$ na população de hipertensos, variando de 20 a 90\%, dependendo de severidade e duração do processo hipertensivo9.

Sendo assim, a DCH tem importância clínica devido à associação com aumento da incidência de insuficiência cardíaca congestiva, arritmia ventricular, infarto do miocárdio, acidente vascular cerebral, doença coronária e a claudicação intermitente, sendo um forte preditor de todas as causas de morte cardíaca em adultos acima de 40 anos, como mostraram os estudos de Framingham. Além disso, evidências sugerem que a regressão da hipertrofia ventricular associa-se à redução da mortalidade cardiovascular ${ }^{3,10}$.

A DCH leva a alterações anátomo-fisiológicas na função cardíaca como consequência da hipertensão arterial sistêmica, evoluindo com remodelamento cardíaco, com ou sem insuficiência cardíaca congestiva e óbito ${ }^{11,12}$. Segundo o Ministério da Saúde, cerca de 50\% dos casos de DCH são diagnosticados aos 55 anos de idade ou mais, atingindo principalmente homens a partir dos 40 anos e as mulheres após a menopausa, quando perdem a proteção estrogênica, ocorrendo variações de prevalência conforme região geográfica ${ }^{13}$.

Para que ocorra promoção de saúde e prevenção dos agravos da HAS e da DCH, a intervenção assistencial requer o conhecimento do impacto epidemiológico destas, sendo necessários estudos de análise da realidade epidemiológica da $\mathrm{DCH}$, no sentido de dimensionar o problema e caracterizá-lo segundo tempo e características populacionais ${ }^{1}$. Sendo assim, nosso objetivo foi realizar uma análise epidemiológica da mortalidade por DCH nas macrorregiões brasileiras.

\section{MATERIAIS E MÉTODOS}

Trata-se de um estudo descritivo de mortalidade de base populacional com dados secundários referentes à população brasileira. No presente estudo foi realizada uma análise comparativa entre as macrorregiões brasileiras: Norte, Nordeste, Centro-Oeste, Sudeste e Sul, utilizando as variáveis: sexo, faixa-etária, ano do óbito, cor/raça e escolaridade.

Para análise da faixa-etária, foram utilizados os dados de população adulta residente e número de óbitos por DCH do Sistema de Informação sobre Mortalidade (SIM) e do Departamento de Informação e Informática do Sistema Único de Saúde (DATASUS) do Ministério da Saúde relacionado às macrorregiões brasileiras e faixas etárias agrupadas (20 a 39, 40 a 59, 60 a 79 e igual ou mais de 80 anos), no período de 2003-2012. Foram excluídas as faixas-etárias menores de 20 anos, devido à baixa prevalência de $\mathrm{DCH}$. Para comparação foram calculados os coeficientes de mortalidade (CM) por DCH.

Para análise da mortalidade por sexo, foram utilizados dados do número de óbitos por DCH do SIM/DATASUS, do Ministério da Saúde, relacionados às macrorregiões brasileiras e sexo masculino e feminino, não sendo incluídos os ignorados e as faixas etárias $<20$ anos, no período de 2003-2012. Investigando a variável raça/cor, foram utilizados os grupos branco, preto, pardo, amarelo, indígena e ignorado, 
das macrorregiões brasileiras e o número de óbitos por DCH do SIM/DATASUS, sendo excluídas as faixas etárias $<20$ anos, avaliando-se no período de 2003-2012.

Para a pesquisa da mortalidade relacionada à escolaridade, foram utilizados os grupos: nenhuma, 1-3 anos, 4-7 anos, 8-11 anos, 12 anos e mais, e número de óbitos por DCH do SIM/DATASUS, relacionados às macrorregiões brasileiras, sendo excluídas as faixas etárias $<20$ anos, avaliando-se no período de 2003-2012. Para análise da mortalidade no decorrer de 10 anos, foram utilizadas as macrorregiões brasileiras e número de óbitos por DCH do SIM/DATASUS, nos anos de 2003-2012.

Os dados foram descritos através de medidas de frequência simples e relativa, além de gráficos e tabelas. Não foi necessário aprovação de Comitê de Ética em Pesquisa por se tratar de dados de domínio público.

\section{RESULTADOS}

Os coeficientes de mortalidade brutos (CMB) por doença cardíaca hipertensiva, para as macrorregiões brasileiras estão descritos na Tabela 1, na qual se observa grande acréscimo em seus valores a partir de 40 a 59 anos. Considerando toda a população adulta o $\mathrm{CMB}$ foi maior na região $\mathrm{NE}$, chamando a atenção o número de 293 óbitos por DCH por 100.000 indivíduos, com idade igual ou maior que 80 anos. Em comparação, a macrorregião que apresentou os menores valores para as faixas-etárias até 79 anos, foi a Sul.
Em relação a variável raça/cor, a DCH acometeu principalmente as pessoas classificadas como pardas nas regiões Norte, Nordeste e Centro-oeste, e a branca nas regiões Sudeste e Sul, provável reflexo do maior predomínio desses fenótipos populacionais nas respectivas regiões. No Sudeste, foi onde houve maior número de óbitos dentre as macrorregiões, com um total de 71.059. O inverso ocorreu na região norte, com 6981 óbitos, como apresentado na Tabela 2.

Analisando a Tabela 3, podemos inferir que o número de óbitos é elevado em ambos os sexos. Para o sexo feminino, os valores foram maiores na região Sudeste, com 38.797, seguida pelo Nordeste com 25.166 e pela região Sul, com 13.132 óbitos, enquanto que no sexo masculino, os maiores valores foram nas regiões Centro-oeste e Norte, com 5.093 e 3.787 óbitos, respectivamente.

Tabela 1. Coeficiente de mortalidade bruto por Doença Cardíaca Hipertensiva em relação à faixa etária e região (dados por 100.000).

\begin{tabular}{ccccc}
\hline \multirow{2}{*}{ Região } & \multicolumn{5}{c}{ Faixa etária (anos) } \\
\cline { 2 - 5 } & $\mathbf{2 0}$ a $\mathbf{3 9}$ & $\mathbf{4 0}$ a $\mathbf{5 9}$ & $\mathbf{6 0}$ a $\mathbf{7 9}$ & $\mathbf{2 8 0}$ \\
$\mathrm{N}$ & 0,23 & 3,78 & 41,12 & 227,42 \\
$\mathrm{NE}$ & 0,45 & 6,42 & 50,78 & 293,40 \\
$\mathrm{SE}$ & 0,46 & 6,83 & 44,91 & 220,98 \\
$\mathrm{~S}$ & 0,17 & 3,72 & 40,51 & 271,11 \\
$\mathrm{CO}$ & 0,38 & 6,38 & 54,12 & 270,49 \\
\hline
\end{tabular}

N: Norte; NE: Nordeste; SE: Sudeste; S: Sul; CO: Centro-Oeste.

Tabela 2. Número de óbitos absolutos por Doença Cardíaca Hipertensiva em relação cor/raça.

\begin{tabular}{ccccccccc}
\hline Região & Branca & Preta & Amarela & Parda & Indígena & Ignorado & Total \\
N & 1574 & 731 & 26 & 4326 & 56 & 268 & 5981 \\
NE & 11979 & 5326 & 165 & 26224 & 88 & 5192 & 38974 \\
SE & 41656 & 9099 & 452 & 16038 & 45 & 26 & 589 & 22526 \\
S & 19105 & 1129 & 66 & 1612 & 47059 & 10173 \\
CO & 4086 & 988 & 49 & 4742 & 54 & 254 & 10071 \\
Total & 78400 & 17273 & 758 & 52942 & 269 & 159713 \\
\hline
\end{tabular}

N: Norte; NE: Nordeste; SE: Sudeste; S: Sul; CO: Centro-Oeste.

Tabela 3. Número absoluto de óbitos por Doença Cardíaca Hipertensiva em relação ao sexo.

\begin{tabular}{cccc}
\hline Região & Masculino & Feminino & Total \\
\hline N & 3787 & 3194 & 6981 \\
NE & 23808 & 25166 & 48974 \\
SE & 32262 & 38797 & 71059 \\
S & 9394 & 13132 & 22526 \\
CO & 5093 & 5080 & 10173 \\
Total & 74344 & 85369 & 159713 \\
\hline
\end{tabular}

N: Norte; NE: Nordeste; SE: Sudeste; S: Sul; CO: Centro-Oeste.
Segundo a escolaridade, percebemos que o número de óbitos é inversamente proporcional à quantidade de anos de escolaridade em todas as regiões. Os maiores valores de mortalidade estão dispostos no grupo de analfabetos, tendo maior evidência na região Nordeste, com 20.078 óbitos, e menor na região Norte com um número de 2.619. Quando analisado o grupo de maior escolaridade, a região Sudeste destaca-se com um valor 14 vezes maior que a região com menor mortalidade, a Norte (Tabela 4). 
Tabela 4. Mortalidade por Doença Cardíaca Hipertensiva em relação à escolaridade.

\begin{tabular}{|c|c|c|c|c|c|c|c|}
\hline Região & Nenhuma & 1 a 3 anos & 4 a 7 anos & 8 a 11 anos & 12 anos e mais & Ignorado & Total \\
\hline N & 2619 & 1751 & 892 & 300 & 120 & 1299 & 6981 \\
\hline NE & 20078 & 9719 & 4301 & 1496 & 538 & 12842 & 48974 \\
\hline SE & 12156 & 17074 & 11647 & 4251 & 1695 & 24236 & 71059 \\
\hline S & 5100 & 7293 & 4256 & 1006 & 396 & 4475 & 22526 \\
\hline $\mathrm{CO}$ & 3216 & 2627 & 1550 & 522 & 187 & 2071 & 10173 \\
\hline Total & 43169 & 38464 & 22646 & 7575 & 2936 & 44923 & 159713 \\
\hline
\end{tabular}

N: Norte; NE: Nordeste; SE: Sudeste; S: Sul; CO: Centro-Oeste. Nenhuma: analfabeto; Ignorado: não declarado.

Notamos que houve aumento progressivo no numero de óbitos nas regiões Norte e Sul, no decorrer destes 10 anos. Já nas regiões Norte e Sudeste, houve aumento progressivo até os anos de 2008 e 2009, e a partir daí ocorreu oscilação no número de óbitos. Analisando a região Centro-Oeste, vemos que houve variação dos valores de 2003 a 2006, com posterior acréscimo no número de óbitos nos anos subsequentes. Percebemos que a maior mortalidade ocorreu na região Sudeste, com um total de 71.059 óbitos, tendo um pico de 7.616 óbitos no ano de 2008, número maior que qualquer outra região atingiu nos 10 anos (Figura 1).

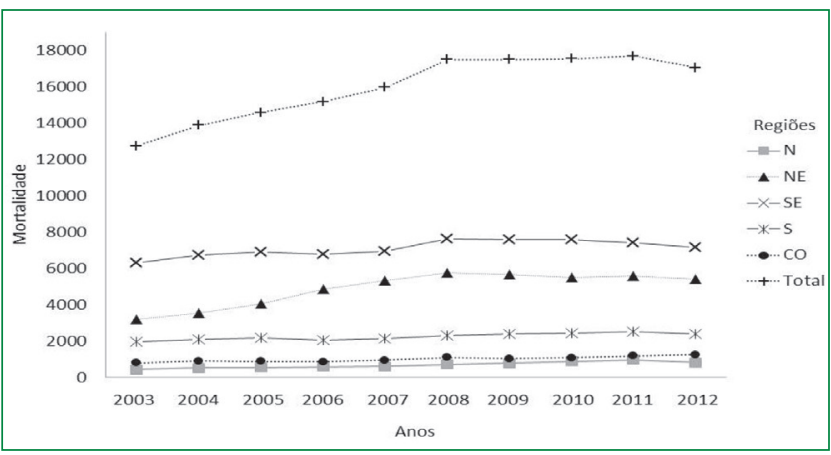

Figura 1. Mortalidade por Doença Cardíaca Hipertensiva no período de 2003-2012.

\section{DISCUSSÃO}

Os resultados sugerem que com o aumento da idade, há elevação do coeficiente de mortalidade, devido a alterações anátomo-fisiológicas que se desenvolvem com o passar dos anos em consequência da HAS. Isto ocorre especialmente onde há maior precariedade na assistência à saúde, nas medidas de prevenção, controle e acompanhamento e repasse de recursos, a exemplo da região Nordeste ${ }^{9}$.

Sabe-se que este quadro é passível de prevenção com a ampliação da triagem e acompanhamento, maiores investimentos no HIPERDIA, controle das complicações, capacitação dos profissionais, diagnóstico precoce dos pacientes de alto risco, dentre outras medidas ${ }^{14}$.
As doenças cardiovasculares (DCV) representam a principal causa de óbitos nos países em desenvolvimento, acometendo a população em idade produtiva. Entretanto, nos idosos, vem apresentando declínio devido à melhoria relativa das condições medico-assistenciais e socioeconômicas ${ }^{1}$. Além disso, tem-se verificado que o número de anos de vida perdidos por conta das DCV de modo geral, vem diminuindo a despeito do aumento da expectativa de vida e à queda da mortalidade infantil ${ }^{15}$.

A hipertrofia ventricular esquerda é fator de risco independente para morbidade e mortalidade cardiovascular ${ }^{16}$. O desenvolvimento de anormalidade estrutural no miocárdio com hipertrofia (desestruturação arquitetural e fibrose) pode predispor a morte súbita em jovens, decorrentes de taquiarritmias ventriculares, nas regiões lesadas ${ }^{17}$. Alguns biomarcadores vêm sendo estudados para determinar o prognóstico em pacientes hipertensos apontando o risco de desenvolver insuficiência cardíaca, a exemplo das citocinas inflamatórias, mais especificamente a Cardiotrofina-1, membro da família da interleucina-6 (IL-6) ${ }^{18}$.

Distúrbios no sistema nervoso autônomo parecem contribuir para a indução de resistência ao tratamento e indução de hipertensão crônica, agravando o remodelamento do ventrículo esquerdo, o que aumenta a pós-carga cardíaca e anormalidades na função diastólica, aumentando o risco de mortalidade e desenvolvimento de insuficiência cardíaca crônica. Além disso, o êxito na redução da hipertrofia ventricular com agentes farmacêuticos, não é tão uniforme, especialmente em pacientes com hipertensão refratária ${ }^{12,19}$.

A necessidade do conhecimento epidemiológico da DCH no Brasil possibilita discriminar as suas as regiões mais acometidas, favorecendo a determinação da magnitude do problema em associação com a faixa-etária, sexo, escolaridade e raça/cor, permitindo intervenções terapêuticas capazes de melhorar o prognóstico clínico e aumentar a sobrevida antes de chegarmos a estes números elevados e progressivos de óbitos ${ }^{3}$.

Observamos que com o aumento da idade, há elevação do coeficiente de mortalidade, especialmente onde há 
maior comprometimento da assistência à saúde e das medidas farmacológicas e não farmacológicas de controle da HAS, a exemplo da região Nordeste. A DCH acometeu principalmente pessoas pardas nas regiões Norte, Nordeste e Centro-oeste e indivíduos brancos nas regiões Sul e Sudeste. Quanto ao sexo, a mortalidade é elevada em ambos. O número de óbitos é inversamente proporcional à quantidade de anos de escolaridade em todas as regiões, sendo os maiores valores no grupo de analfabetos.

\section{REFERÊNCIAS}

1. Godoy MF, Lucena JM, Miquelin AR, Paiva FF, Oliveira DL, Augustin Junior JL, Chiaravalloti Neto F. Cardiovascular mortality and its relation to socioeconomic levels among inhabitants of Sao Jose do Rio Preto, Sao Paulo state, Brazil. Arq Bras Cardiol. 2007;88(2): 200-6. https://doi.org/10.1590/S0066-782X2007000200011

2. Malachias MVB, Souza WKSB, Plavnik FL, Rodrigues CIS, Brandão AA, Neves MFT, Bortolotto LA, Franco RJS, Poli-de-Figueiredo CE, Jardim PCBV, Amodeo C, Barbosa ECD, Koch V, Gomes MAM, Paula RB, Póvoa RMS, Colombo FC, Ferreira Filho S, Miranda RD, Machado CA, Nobre F, Nogueira AR, Mion Júnior D, Kaiser S, Forjaz CLM, Almeida FA, Martim JFV, Sass N, Drager LF, Muxfeldt E, Bodanese LC, Feitosa AD, Malta D, Fuchs S, Magalhães ME, Oigman W, Moreira Filho O, Pierin AMG, Feitosa GS, Bortolotto MRFL, Magalhães LBNC, Silva ACS, Ribeiro JM, Borelli FAO, Gus M, Passarelli Júnior O, Toledo JY, Salles GF, Martins LC, Jardim TSV, Guimarães ICB, Antonello IC, Lima Júnior E, Matsudo V, Silva GV, Costa LS, Alessi A, Scala LCN, Coelho EB, Souza D, Lopes HF, Gowdak MMG, Cordeiro Júnior AC, Torloni MR, Klein MRST, Nogueira PK, Lotaif LAD, Rosito GBA, Moreno Júnior H. 7a Diretriz Brasileira de Hipertensão Arterial. Arq Bras Cardiol. 2016;107 (3 Supl 3):1-83. https://doi.org/10.5935/abc.20160140

3. Lessa I. Epidemiologia da hipertensão arterial sistêmica e da insuficiência cardíaca no Brasil. Rev Bras Hipertens. 2001;8:383-92.

4. Sant'Anna MP, Mello RJVd, Montenegro LT, Araújo MM. Left and right ventricular hypertrophy at autopsy of hypertensive individuals. Rev Assoc Med Bras. 2012;58(1):41-7. https://doi.org/10.1016/ S0104-4230(12)70153-7

5. McMaster, WG. Kirabo, A. Madhur, MS, Harrison, DG. Inflammation, Immunity, and Hypertensive End-Organ Damage. Circ Res. 2015;116(6):1022-33. https://doi.org/10.1161/CIRCRESAHA.116. 303697

6. Acharya T, Tringali S, Singh M, Huang J. Resistant hypertension and associated comorbidities in a veterans affairs population. J Clin Hypertens (Greenwich). 2014 Oct;16(10):741-5. https://doi. org/10.1111/jch.12410

7. Nogueira PR, Rassi S, Corrêa KdS. Epidemiological, clinical e therapeutic profile of heart failure in a tertiary hospital. Arq Bras Cardiol. 2010;95(3):392-8. https://doi.org/10.1590/S0066782X2010005000102

8. Bocchi EA, Marcondez-Braga FG, Bacal F, Ferraz AS, Albuquerque D, Rodrigues D, Mesquita ET, Vilas-Boas F, Cruz F, Ramirez F, Villacorta
Junior H, Souza Neto JD, Rossi Neto JM, Moura LZ, Beck-da-Silva L, Moreira LF, Rohde LEP, Montera MW, Simões MV, Moreira MC, Clausell N, Bestetti R, Mourilhe-Rocha R, Mangini S, Rassi S, AyubFerreira SM, Martins SM, Bordignon S, Issa VS. Sociedade Brasileira de Cardiologia. Atualização da Diretriz Brasileira de Insuficiência Cardíacacrônica - 2012. Arq Bras Cardiol. 2012;98(1 Supl. 1):1-33. https://doi.org/10.1590/S0066-782X2012001000001

9. Raman SV. The Hypertensive heart: an integrated understanding informed by imaging. J Am Coll Cardiol. 2010;55(2):91-6. https:// doi.org/10.1016/j.jacc.2009. 07.059

10. Bombig MTN, Povoa R. Cardiopatia hipertensiva: aspectos epidemiológicos, prevalência e fator de risco cardiovascular. Rev Bras Hipertens. 2008;15(2):75-80.

11. Hoey ETD, Pakala V, Teoh JK, Simpson H. The Role of Imaging in Hypertensive Heart Disease. Int J Angiol. 2014 Jun;23(2):85-92. https://doi.org/10.1055/s-0034-1370885

12. Lai YH, Lo $\mathrm{Cl}, \mathrm{Wu} \mathrm{YJ}$, Hung $\mathrm{CL}$, Yeh HI. Cardiac Remodeling, Adaptations and Associated Myocardial Mechanics in Hypertensive Heart Diseases. Zhonghua Minguo Xin Zang Xue Hui Za Zhi. 2013 Jan;29(1):64-70.

13. Schmidt A. Insuficiência cardíaca diastólica e sistólica em pacientes hipertensos: diagnóstico e tratamento diferenciais Revista Brasileira de Hipertansão. Rev Bras Hipertens. 2001;8:440-44.

14. Schmidt MI, Duncan BB, Hoffmann JF, Moura Ld, Malta DC, Carvalho RMSVd. Prevalence of diabetes and hypertension based on self-reported morbidity survey, Brazil, 2006. Rev Saúde Públ. 2009;43:74-82. https://doi.org/10.1590/S003489102009000900010

15. Simöes CCdS. Perfis de saúde e de mortalidade no Brasil: uma análise de seus condicionantes em grupos populacionais específicos. Brasília: OPAS; 2002.

16. Antoniucci D, Seccareccia F, Menotti A, Dovellini EV, Prati PL, Rovelli F, Fazzini PF. Prevalence and correlates of echocardiographic determined left ventricular hypertrophy in 2318 asymptomatic middle-aged men: the ECCIS project. Epidemiolgia e Clinica della Cardiopatia Ischemica Silente. G Ital Cardiol. 1997;27(4): 363-9.

17. Chan RH, Maron BJ, Olivotto I, Pencina MJ, Assenza GE, Haas T, Lesser JR, Gruner C, Crean AM, Rakowski H, Udelson JE, Rowin E, Lombardi M, Cecchi F, Tomberli B, Spirito P, Formisano F, Biagini E, Rapezzi C, De Cecco CN, Autore C, Cook EF, Hong SN, Gibson CM, Manning WJ, Appelbaum E, Maron MS. Prognostic value of quantitative contrast-enhanced cardiovascular magnetic resonance for the evaluation of sudden death risk in patients with hypertrophic cardiomyopathy. Circulation. 2014;130(6):484-95. https://doi. org/10.1161/CIRCULATIONAHA.113.007094

18. Song K, Wang S, Huang B, Luciano A, Srivastava R, Mani A. Plasma cardiotrophin-1 levels are associated with hypertensive heart disease: a meta-analysis. J Clin Hypertens (Greenwich). 2014;16(9):686-92. https://doi.org/10.1111/jch.12376

19. Todoran TM, Zile MR. Neuromodulation device therapy for treatment of hypertensive heart disease. Circ J. 2013;77(6): 1351-63. https://doi.org/10.1253/circj.CJ-13-0390 